\title{
Influencia de rhBMP-2 en la Reparación Ósea de Defectos Críticos con Diferentes Biomateriales
}

\author{
Influence of rhBMP-2 on Bone Repair of Critical Size Defects with Different Biomaterials
}

\author{
Francisca Uribe ${ }^{1,2}$; Bélgica Vásquez ${ }^{3}$; Carlos Veuthey ${ }^{4}$; Juan Pablo Alister ${ }^{2}$ \& Sergio Olate Se, $^{2,4}$
}

URIBE, F.; VÁSQUEZ, B.; VEUTHEY, C.; ALISTER, J. P. \& OLATE, S. Influencia de rhBMP-2 en la reparación ósea de defectos críticos con diferentes biomateriales. Int. J. Morphol., 38(2):316-321, 2020.

RESUMEN: La regeneración de defectos óseos críticos requiere la utilización de biomateriales óseos. Así, se han utilizados agentes osteogénicos como la proteína morfogenética (rhBMP-2). El objetivo fue describir la formación ósea de defectos óseos críticos en calota de ratas utilizando rhBMP-2 con distintos biomateriales. Se realizaron dos defectos óseos críticos de $5 \mathrm{~mm}$ en 15 calotas de ratas machos adultas divididos en grupo control (sin tratamiento) (C); autoinjerto + rhBMP-2 (A); fosfato tricálcico + rhBMP-2 (BTCP); xenoinjerto de bovino + rhBMP-2 (B) y hidroxihapatita + rhBMP-2 (HA). A las ocho semanas post tratamiento, se realizó la eutanasia y posterior análisis histológico de los defectos. El grupo C no presentó formación de tejido óseo en el defecto. En el resto de los grupos, se formó abundante tejido óseo en los márgenes, por lo tanto, el defecto presentó menor tamaño. El grupo HA presentó formación ósea trabecular con amplios espacios medulares y abundante tejido adiposo. El grupo B-TCP también presentó formación ósea trabecular y la mayoría de las muestras presentaron puente óseo en el defecto. El grupo B presentó partículas de material injertado rodeado por trabéculas óseas y tejido conectivo. En el grupo A, todas las muestras presentaban puente óseo formado por bloques de autoinjerto rodeado por tejido conectivo y óseo. Es posible concluir que los defectos óseos de $5 \mathrm{~mm}$ en calota de rata son defectos críticos que requieren utilizar biomateriales para la reparación del defecto. El grupo B-TCP presentó características histológicas más próximas a la regeneración ósea lograda con el Grupo A.

PALABRAS CLAVE: BMP; rhBMP-2; Proteína morfogenética; Regeneración ósea; Biomateriales.

\section{INTRODUCCIÓN}

La regeneración de defectos óseos críticos requiere la utilización de biomateriales que permitan la instalación de una secuencia que lleve a la reparación ósea (SchwartzArad et al., 1997; Zeren, 2006). Históricamente, el autoinjerto ha sido el gold standard debido a sus propiedades osteoconductoras, osteoinductoras y osteogénicas. Sin embargo, el mayor tiempo quirúrgico, morbilidad y potenciales complicaciones, hacen necesario buscar nuevas estrategias para la regeneración ósea (Olate et al., 2019).

El mercado actual ha entregado muchas opciones y variedades de productos que son empleados como sustitutos óseos en cirugías reconstructiva dento-alveolar, tales como los tipos aloplásticos, xenogénicos u homogénicos, los cuales han demostrado resultados adecuados en algunos casos (Vandeweghe et al., 2013; Tovar et al., 2014). Sin embargo, en situaciones de reconstrucciones de alta exigen- cia, los sustitutos óseos no son totalmente útiles, siendo controversial su capacidad en neoformación ósea.

Variables generales como edad del sujeto y el metabolismo óseo, junto a variables locales como la extensión del defecto, calidad de hueso receptor y características del tejido blando en el cierre de la herida, juegan un papel importante en este escenario (Green et al., 2005); casos complejos de mayor necesidad en la técnica reconstructiva requieren que la osteoconducción de algunos biomateriales pueda ser mejorada a través de la osteoinducción de algunos factores de crecimiento como la proteína ósea morfogenética (BMP) (Uribe et al., 2019).

Las BMP son un conjunto de proteínas endógenas que pertenecen a la familia de factor de crecimiento transformante beta (TGF-b) que tienen la capacidad de in-

\footnotetext{
${ }^{1}$ Doctorado en Ciencias Médicas, Facultad de Medicina, Universidad de La Frontera, Temuco, Chile.

${ }^{2}$ División de Cirugía Oral y Maxilofacial, Facultad de Odontología, Universidad de La Frontera, Temuco, Chile.

${ }^{3}$ Facultad de Ciencias de la Salud, Universidad de Tarapacá, Arica, Chile.

${ }^{4}$ Centro de Excelencia en Estudios Morfológicos y Quirúrgicos (CEMyQ), Universidad de La Frontera, Temuco, Chile.
} 
ducir hueso, cartílago y tejido conectivo (Carreira et al., 2014a). La actividad biológica de la BMP es a través de estímulos que desencadenan una formación ósea endocondral. De esta manera, la matriz portadora de BMP hacia el sitio del receptor es fundamental, ya que se asocia a la concentración de BMP que está disponible (Li \& Wozney, 2001).

Actualmente se encuentra aprobado el uso de la proteína morfogenética recombinante humana tipo 2 (rh-BMP2) por la FDA con una matriz de esponja de colágeno, sin embargo, esta matriz presenta desventajas, tales como, una liberación rápida de la rhBMP-2 y una reabsorción acelerada en comparación con la formación ósea (Carreira et al., 2014b). Además, para obtener buenos resultados y generar tejido óseo se utilizan dosis suprafisiológicas, lo que conlleva a complicaciones como edema, eritema, dolor o incluso infección.

Una matriz ideal debe ser biocompatible, biodegradable de una manera predecible, resistente a la tensión y a la compresión, esterilizable y fácil de manipular. El objetivo de la matriz es transportar y mantener la rhBMP-2 en el defecto óseo, promoviendo el reclutamiento de células mesenquimáticas y posterior diferenciación de las células osteoprogenitoras.

Entendiendo que algunos biomateriales presentan adecuadas características en la conducción ósea, el objetivo de esta investigación fue describir la formación ósea de defectos óseos críticos en calota de ratas utilizando rhBMP-2 con distintos biomateriales.

\section{MATERIAL Y MÉTODO}

Se realizó un estudio experimental en modelo animal con 15 ratas Sprague dawley machos, de 16 semanas, sanos y sexualmente maduros, con un peso de 375 a $500 \mathrm{~g}$, aproximadamente. Las ratas fueron mantenidas en condiciones de humedad, temperatura y luz estándar, alimentadas con pellet comercial y agua ad libitum durante todo el experimento. El proyecto de investigación, junto al protocolo de trabajo, fue aprobado por el Comité Ético Científico de la Universidad de La Frontera ( $\mathrm{N}^{\circ}$ Folio 009/17).

Se utilizó rhBMP-2 derivada de Escherichia coli (NOVOSIS-Dent, CGBio Inc., Seongnam, Korea). Los biomateriales utilizados como matriz para la administración conjunta con rhBMP-2 fueron; 1 ) aloinjerto $\beta$-fosfato tricálcico ( $\beta$-TCP) (Excelos BioAlpha Inc, Korea), 2) xenoinjerto bovino (Bio-Oss, Geistlich, Switzerland, 0,25 $1 \mathrm{~mm}$ ) y 3 ) aloinjerto hiodroxiapatita (HA) (IngeniOS HA, Zimmer Dental Inc, USA 0,25 - $1 \mathrm{~mm}$ ).
La dosis de rhBMP-2 utilizada en cada defecto fue de $5 \mu \mathrm{g}$ de rhBMP-2. Se diluyeron $0,25 \mu \mathrm{g}$ de rhBMP-2 en 2,5 $\mathrm{ml}$ de suero fisiológico, obteniendo las dosis adecuadas para la administración en cada defecto. La dosis de rhBMP2 fue mezclada con el material de instalación durante 10 minutos para después ser aplicado en el defecto.

En cada rata se realizaron dos defectos óseos críticos de $5 \mathrm{~mm}$ de diámetro en cada calota. Los grupos fueron distribuidos al azar en los 30 defectos óseos de los animales. Cada animal fue marcado con un número en la cola con lápiz indeleble. En una tabla fueron anotados cada defecto y el grupo de pertenencia, luego fueron ocultados hasta después de obtener los resultados. Los defectos óseos se dividieron en 5 grupos: grupo control (sin tratamiento) (C); autoinjerto + rhBMP-2 (A); $\beta$-TCP + rhBMP-2 (B-TCP); xenoinjerto bovino + rhBMP-2 (B) y HA + rhBMP-2 (HA). En cada grupo se incluyeron 5 defectos óseos los cuales fueron analizados a las 8 semanas de realizada la cirugía experimental.

La cirugía para cada defecto fue realizada bajo anestesia mediante ketamina $80 \mathrm{mg} / \mathrm{kg}$ y xilacina $10 \mathrm{mg} / \mathrm{kg}$ intraperitoneal (CONICYT, 2009). Antes de iniciar el procedimiento se realizó el rasurado y antisepsia con povidona yodada junto a infiltración con lidocaína $2 \%$ + epinefrina $1: 100.000$ diluida a $0,5 \%$ con una dosis máxima de lidocaína de $7 \mathrm{mg} / \mathrm{kg}$ (CONICYT). Se realizó una incisión longitudinal sobre la línea media sagital en la piel de la calota desde el hueso frontal al occipital. Se efectuó la elevación del colgajo de espesor total, exponiendo el tejido óseo para realizar dos defectos óseos bi-corticales mediante trefinas de $5 \mathrm{~mm}$ de diámetro externo, bajo irrigación continua para disminuir el calor producido por el corte, cuidando no dañar la duramadre. Se verificó la hemostasia e integridad de la duramadre y se aplicó el material seleccionado (Fig. 1). La reposición del periostio y sutura se realizó con vicryl 4-0, mientras que en piel se utilizó nylon 4-0. En esta investigación la eutanasia de los animales se realizó a las 8 semanas, utilizando una sobredosis de ketamina $160 \mathrm{mg} / \mathrm{kg}$ y xilacina $20 \mathrm{mg} / \mathrm{kg}$. Los experimentos se llevaron a cabo de acuerdo con las directrices de la Guía para el Cuidado y Uso de Animales de Laboratorio (2011).

Las muestras de calota fueron obtenidas mediante osteotomías realizadas con sistema piezo eléctrico (Piezotome, Acteon Satelec, Francia); posteriormente fueron fijadas en formalina tamponada al $10 \%$, $\mathrm{pH} 7,4$, por 48 horas, descalcificadas en EDTA tamponado al $10 \%$ por un período de tres meses, deshidratados en una batería de alcoholes ascendentes e incluidos en parafina (Histosec, Merck). Se realizaron cortes en sentido coronal de $5 \mu \mathrm{m}$ de grosor en un micrótomo (Microm HM 325, Thermo Fisher 
Scientific Inc., Waltham, MA, USA) y las láminas histológicas fueron teñidas con Hematoxilina \& Eosina (HE) para su análisis. La visualización de los cortes se realizó con un microscopio de luz Leica ${ }^{\circledR}$ DM2000 LED y la fotografía con una cámara digital Leica® MC170 HD.

\section{RESULTADOS}

Tejido óseo de calota normal. El tejido óseo de calota de rata estaba formado principalmente por tejido óseo compacto, revestido por una membrana perióstica de tejido conectivo que contenía vasos sanguíneos que atravesaban el tejido óseo, asegurando la irrigación del hueso. El tejido óseo compacto estaba compuesto por lamelas óseas dispuestas paralela a la superficie, con múltiples osteocitos ubicados en sus lagunas y por lamelas concéntricas de las osteonas. En la zona media de la calota, se observaban osteonas cuyo canal central era de mayor diámetro, lo que permitía mayor vascularización de la zona (Fig. 2).

Grupo control (C) (sin tratamiento). El límite entre el tejido óseo preexistente y el tejido óseo formado era evidente. En los márgenes del defecto, se observaban superficies de osificación con reducción del tamaño del defecto. La zona central del defecto no presentó tejido óseo, se caracterizó por la presencia de tejido conectivo laxo, con presencia de vascularización y celularidad. En la zona superficial del defecto, las fibras colágenas eran de mayor densidad y presentaban una disposición más uniforme que en la zona profunda (Fig. 3A).

Grupo autoinjerto + rhBMP-2 (A). El límite entre el tejido óseo pre-existente y el tejido óseo formado era definido. En la mayoría de las muestras se observó un cierre total del defecto óseo, no obstante, en algunas aún presentaban remanentes de bloques de autoinjerto. El tejido óseo formado era de tipo compacto que se caracterizaba por presentar lamelas aun desorganizadas con presencia de pequeños espacios medulares ubicados principalmente en la zona media, entre la zona superficial y profunda. El tejido formado en el defecto no logró el mismo grosor que el hueso parietal, mientras que el periostio se encontraba organizado en la periferia con abundantes vasos sanguíneos (Fig. 3B).

Grupo b-TCP + rhBMP-2 (B-TCP). El límite entre el tejido óseo preexistente y el tejido óseo formado es definido. El grosor del tejido formado en el defecto era menor al grosor del hueso parietal. La mayoría de las muestras presentaron puente óseo. El tejido óseo formado próximo a los márgenes del defecto era de tipo compacto cuyas lamelas aún estaban desorganizadas; el hueso formado en el centro del defecto era de tipo trabecular con amplios espacios medulares. En las muestras que no presentaban puente óseo, el tejido conectivo mostraba abundantes fibras colágenas, y en algunos sectores se observó colágeno calcificado dispuestos alrededor de pequeñas trabéculas (Fig. 3C).

Grupo xenoinjerto bovino + rhBMP-2 (B). El límite entre el tejido óseo pre-existente y el tejido óseo formado era definido. El grosor del tejido formado en el defecto era menor al grosor del hueso parietal. Todas las mues- tras presentaron en la zona del defecto partículas del material injertado. En algunas muestras estas partículas se encontraban rodeadas por trabéculas óseas anguladas, ya que la disposición espacial de éstas se relacionaban con la forma del material instalado. El tejido conectivo presentaba predominio de colágeno y fibroblastos y era más escaso entre las trabéculas. En algunas muestras el puente estaba formado sólo por partículas de material injertado rodeado por tejido conectivo (Fig. 3D).

Grupo HA + rhBMP-2 (HA). El límite entre el tejido óseo pre-existente y el tejido óseo formado estaba bien definido. En los márgenes del defecto, se observó superficie de osificación presentando un defecto de menor tamaño. El grosor del tejido formado en el defecto era menor al grosor del hueso parietal. Al centro del defecto presentaba tejido conectivo organizado y en los espacios medulares en formación tejido adiposo (Fig. 3E).

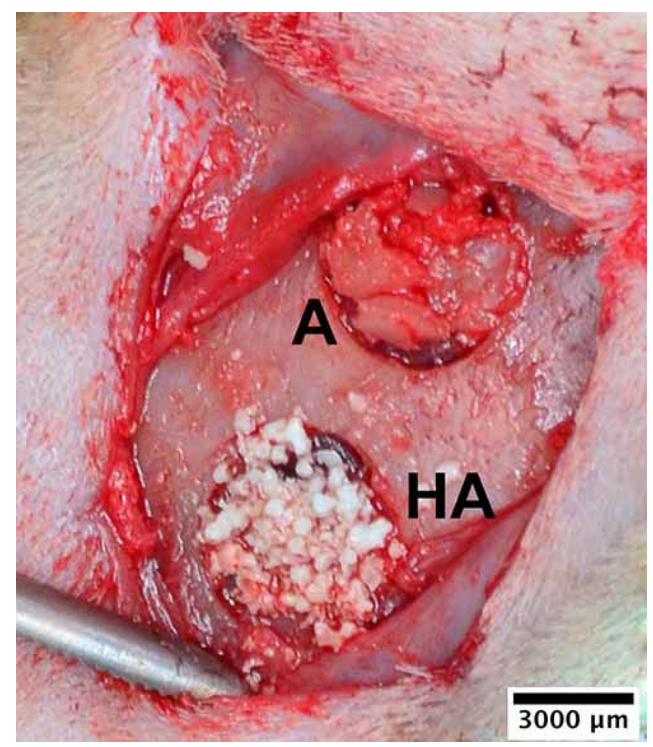

Fig. 1. Defecto de $5 \mathrm{~mm}$ de diámetro realizado en calota de rata Sprague dawley. HA. Defecto con hidroxiapatita. A. Defecto con autoinjerto.

\section{DISCUSIÓN}

El defecto crítico es un modelo utilizado en diferentes investigaciones para analizar la eficiencia de algunos materiales. El uso de ratas presenta la ventaja de ser un modelo de fácil manejo, bajo costo y de uso confirmado 

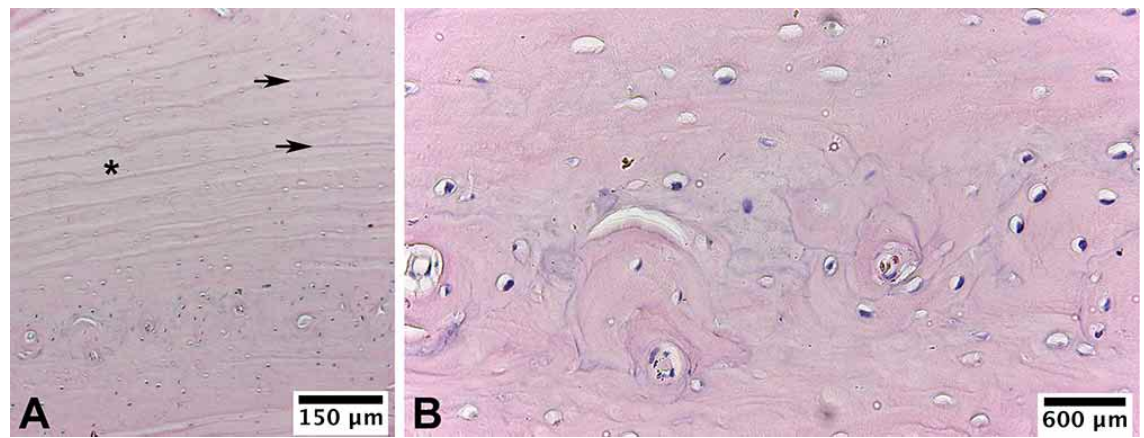

Fig. 2. Tejido óseo de calota de rata Sprague dawley. A. Se observaba tejido óseo compacto $(*)$ y lamelas óseas $(\rightarrow)$ dispuestas paralela a la superficie. B. En la zona media se observan osteonas, cuyos canales centrales permiten la presencia de vasos sanguíneos.
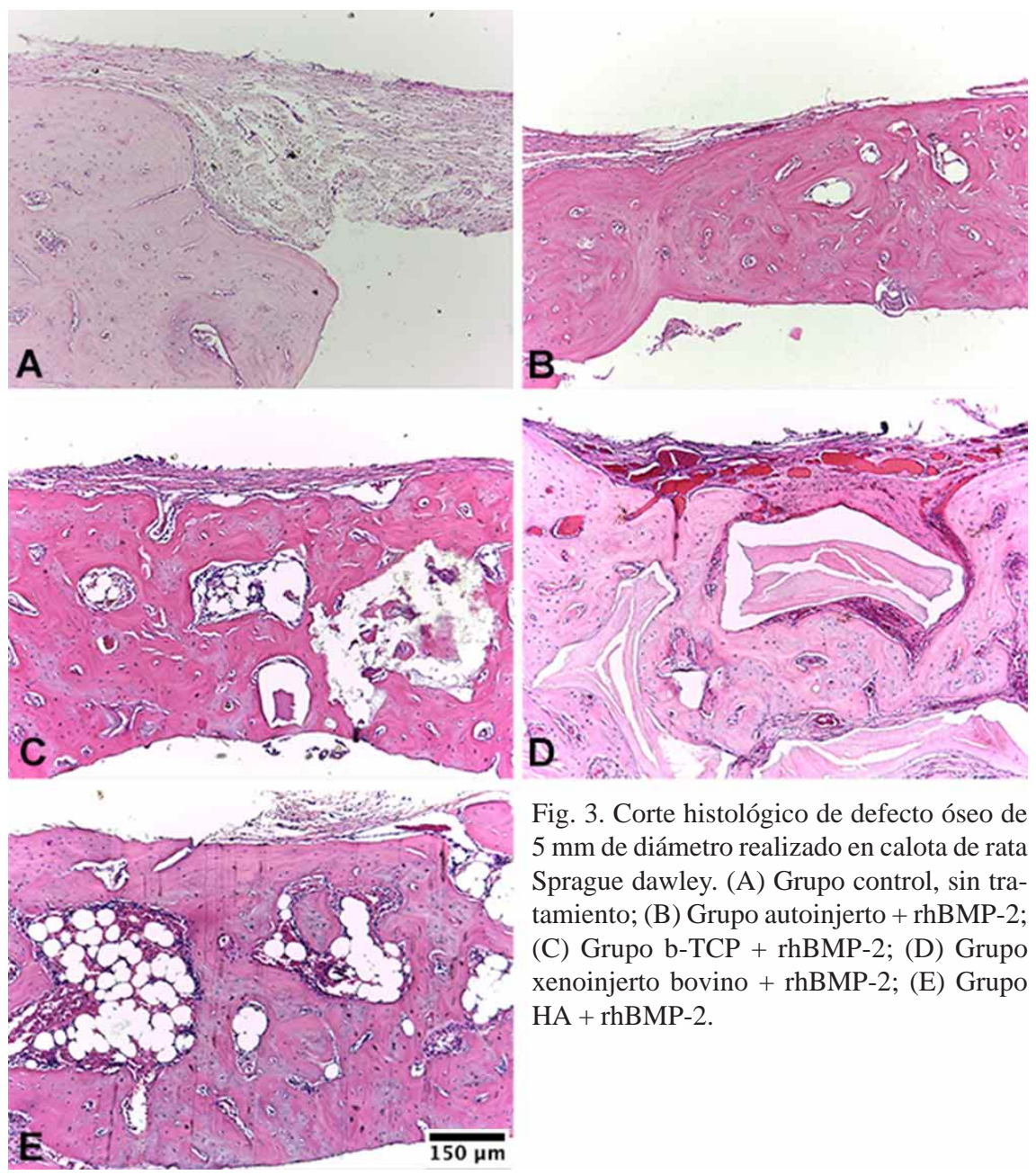

Fig. 3. Corte histológico de defecto óseo de $5 \mathrm{~mm}$ de diámetro realizado en calota de rata Sprague dawley. (A) Grupo control, sin tratamiento; (B) Grupo autoinjerto + rhBMP-2; (C) Grupo b-TCP + rhBMP-2; (D) Grupo xenoinjerto bovino + rhBMP-2; (E) Grupo $\mathrm{HA}+$ rhBMP-2.

para evaluar la regeneración ósea. Algunos requisitos para el adecuado desarrollo de la reparación ósea incluyen el cierre primario del tejido blando, presencia de angiogénesis que permita la irrigación sanguínea de los tejidos, presencia de células mesenquimales no diferenciadas, mantención adecuada del lecho quirúrgico y estabilidad de la región en re- paración junto al material instalado (si fuesen utilizados injertos) (Wang \& Boyapati, 2006). El tipo de defecto óseo define en gran parte la forma física del injerto a ser utilizado, intentando un posicionamiento adecuado junto a la disminución de espacios entre el injerto y el lecho receptor (Nishimura et al., 2004).

El defecto crítico generado en este modelo fue confirmado, dado que en el grupo control no se observó formación ósea. Por otra parte, los biomateriales utilizados en esta investigación han sido utilizados en otras investigaciones y procedimientos clínicos, por lo tanto, este estudio se presenta como un análisis al desarrollo actual de la cirugía de reconstrucción del proceso alveolar maxilar y mandibular.

La proteína ósea morfogenética es uno de los factores de crecimiento que tiene la ventaja de ser un potente inductor óseo (Freitas et al., 2015); la aplicación desarrollada en cirugía maxilofacial y en implantología oral ha sido exitosa en su mayoría, aunque las técnicas específicas para aumentos óseos de la zona alveolar han sido limitadas (Uribe et al., 2017). Teóricamente, la presencia del material osteoconductor (matriz) y el osteoinductor (factor de crecimiento rhBMP-2) tendrían una sinergia para la formación ósea en diferentes lugares del defecto; sin embargo, nuestros resultados iniciales muestran una formación ósea principalmente desde la periferia hacia el centro del defecto, lo que limita la potencialidad de los hallazgos observados en esta investigación.

rhBMP-2 y rhBMP-7 puede acelerar la formación ósea (Uribe et al., 2017), pero también puede acelerar la inducción para la formación de osteoclastos asociados a la reabsorción prematura de hueso mediante activación y señalización de RANKL-RANK, lo cual ha sido confirmado parcialmente en algunos estudios (Kaneko et al., 2000; 
Seeherman et al., 2010). Esto podría justificar algunos resultados inciertos presentados en algunas publicaciones sobre la aplicación clínica de rhBMP-2, como el de Raina et al. (2018), quienes demostraron que rhBMP-2 indujo la reabsorción ósea prematura basada en osteoclastos, lo que podría reducir la formación ósea total al ser comparada con otros modelos de instalación de rhBMP-2. En esta investigación se han observado resultados descriptivos que indican estados de formación ósea avanzada con diferentes matrices de rhBMP-2 en algunos grupos, de forma que aquellos que presentaron menor nivel de reparación pudieron ser influenciados con una reabsorción ósea precoz asociada a estos fenómenos de formación de osteoclastos. En esta dirección, la matriz de la rhBMP-2 juega un rol importante ya que su velocidad de degradación genera un espacio mayor para la neoformación ósea.

Por otra parte, estudios preclínicos realizados indican una relación inversa dosis-efecto, lo que implica que la aplicación de una dosis superior a la óptima de rhBMP-2 (no confirmada en humanos o modelos animal), puede influir negativamente en la formación ósea, retrasando la maduración de la misma (Wikesjö et al., 2008 a y b). Esta variable también pudo influenciar las observaciones realizadas en esta investigación, donde el hueso autógeno se presentó como el material de mejor rendimiento cuando se analizaron en conjunto con los otros tipos de materiales.

La matriz utilizada para la aplicación de rhBMP-2 tiene relevancia para la estabilización ósea en formación y para conseguir resultados clínicamente adecuados (Raina et al.; Alister et al., 2019); de esta manera, el vehículo osteoconductor que permita actuar como matriz para la regeneración ósea debe ser adecuadamente valorado, de forma que entregue estabilidad mecánica y permita la regeneración en el sitio.

Naujokat et al. (2019) estudiaron la formación ectópica de hueso en tejido muscular en 3 grupos, utilizando cubos de HA bovina, un cubo bifásico de HA y tricalcio fosfato $\mathrm{y}$, un cubo a base de titanio impreso; en ellos se realizaron las perforaciones para instalar una membrana de colágeno con células proveniente de cresta iliaca y rhBMP-2; ocho semanas después, en cirugía de reentrada se instalaron los implantes en los bloques. En todos los casos existió formación de hueso, aunque el bloque de origen bovino mostró la menor cantidad en formación ósea. En ese sentido, nuestros resultados señalan prematuramente que el biomaterial de origen bovino presentaría persistencia de las partículas del material y consecuentemente menor formación ósea, lo cual debe confirmarse con futuras investigaciones. Otros autores han demostrado resultados variables en términos de formación ósea cuando utilizaron mezclas de rhBMP-2 con material óseo particulado de origen bovino (Jung et al., 2003; Kao et al., 2012), lo cual puede estar asociado a la matriz para aplicar la rhBMP-2.
La porosidad que puedan presentar estos biomateriales también juegan un rol importante ya que la migración celular a través de los canalículos se vería afectada cuando estos son muy pequeños, disminuyendo la opción de flujo vascular, mientras que cuando aumenta mucho en tamaño, disminuye la resistencia mecánica del material (Alister et al.,). De la misma manera, el tamaño de las partículas de los biomateriales tienen un protagonismo importante, ya que tamaños menores de partículas de biomaterial contribuyen a una mayor formación ósea (Klüppel et al., 2013), entregando mayor versatilidad a las técnicas reconstructivas. Estos elementos influyen en la reparación de defectos, donde la rhBMP-2 puede ser un factor positivo para la neoformación ósea.

\section{CONCLUSIÓN}

Es posible concluir que todos los materiales utilizados en esta investigación en conjunto con rhBMP-2 son útiles para la reparación ósea; sin embargo, el hueso autógeno muestra un avanzado estado de reparación, mientras que otros materiales como el bovino se encuentran con persistencia de partículas dentro del defecto, limitando la regeneración de tejido óseo. El puente óseo fue observado en casi todos los grupos, sin embargo, la formación ósea distante de la periferia del defecto siempre fue menor, demostrando una limitada capacidad de establecer diferentes centros de osificación. Un análisis de mayor extensión debe ser realizado para entender de mejor forma el rol de la rhBMP-2 junto a diferentes biomateriales.

\section{AGRADECIMIENTOS}

Los autores agradecen a la Dirección de Investigación de la Universidad de La Frontera por su financiamiento a través del proyecto DIUFRO DI19-0047. La autora Francisca Uribe Fenner agradece a La Comisión Nacional de Investigación Científica y Tecnológica, CONICYT, por financiar sus estudios de Doctorado CONICYT-PCHA/ doctoradonacional/2015-21150752.

URIBE, F.; VÁSQUEZ, B.; VEUTHEY, C.; ALISTER, J. P. \& OLATE, S. Influence of rhBMP-2 on bone repair of critical defects with different biomaterials. Int. J. Morphol., 38(2):316-321, 2020.

SUMMARY: The regeneration of bone critical size defects requires the use of bone biomaterials. Therefore, an osteogenic agent such as bone morphogenetic protein (rhBMP-2) has been used. The objective was to describe the bone formation of bone critical size defects in the rat calvaria using rhBMP-2 with different biomaterials. Two 
critical bone defects of $5 \mathrm{~mm}$ were made in 15 calvaria of adult male rats divided into a control group (without treatment) (C); autograft + rhBMP-2 (A); tricalcium phosphate + rhBMP-2 (B-TCP); bovine xenograft + rhBMP-2 (B) and hydroxyhapatite + rhBMP-2 (HA). At eight weeks post treatment, euthanasia and subsequent histological analysis of the defects were performed. Group $\mathrm{C}$ did not show bone tissue formation in the defect. In the rest of the groups, abundant bone tissue formed in the margins, therefore, the defect was smaller. The HA group presented trabecular bone formation with large medullary spaces and abundant adipose tissue. The B-TCP group also presented trabecular bone formation and most of the samples formed a bone bridge across the defect. Group B presented grafted material particles surrounded by bone trabeculae and connective tissue. In group A, all samples presented a bone bridge formed by autograft blocks surrounded by connective and bone tissue. It is possible to conclude that $5 \mathrm{~mm}$ bone defects in rat calvaria are critical size defects that require the use of biomaterials for defect repair. The B-TCP group presented histological characteristics similar to the bone regeneration achieved with Group A.

KEY WORDS: BMP; rhBMP-2; Morphogenetic protein; Bone regeneration; Biomaterials.

\section{REFERENCIAS BIBLIOGRÁFICAS}

Alister, J. P.; Uribe, F.; Vásquez, B.; Fariña, R. \& Olate, S. Characterization of bone substitute b-TCP block for maxillary reconstruction. Int. J. Morphol., 37(1):82-6, 2019.

Carreira, A. C.; Alves, G. G.; Zambuzzi, W. F.; Sogayar, M. C. \& Granjeiro, J. M. Bone morphogenetic proteins: structure, biological function and therapeutic applications. Arch. Biochem. Biophys., 561:64-73, 2014a.

Carreira, A. C.; Lojudice, F. H.; Halcsik, E.; Navarro, R. D.; Sogayar, M. C.; Granjeiro, J. M. Bone morphogenetic proteins: facts, challenges, and future perspectives. J. Dent. Res., 93(4):335-45, 2014b.

Comisión Nacional de Investigación Científica y Tecnológica. Aspectos bioéticos de la experimentación animal. Comité Asesor de Bioética, FONDECYTCONICYT, Santiago, Chile, 2009. p. 140.

Committee for the Update of the Guide for the Care and Use of Laboratory Animals; Institute for Laboratory Animal Research (ILAR); Division on Earth and Life Studies (DELS); National Research Council. (8 Ed.). Guide for the Care and Use of Laboratory animals. Washington, DC, The National Academies Press, 2011.

Freitas, R. M.; Spin-Neto, R.; Marcantonio Junior, E.; Pereira, L. A.; Wikesjö, U. M. \& Susin, C. Alveolar ridge and maxillary sinus augmentation using rhBMP-2: A systematic review. Clin. Implant Dent. Relat. Res., 17(Suppl 1):e192-201, 2015

Green, E.; Lubahn, J. D. \& Evans, J. Risk factors, treatment and outcomes associated with nonunion of the midshaft humerus fracture. J. Surg. Orthop. Adv., 14(2):64-72, 2005.

Jung, R. E.; Glauser, R.; Schärer, P.; Hammerle, C. H.; Sailer, H. F. Weber, F. E. Effect of rhBMP-2 on guided bone regeneration in humans. Clin. Oral Implant Res., 14(5):556-68, 2003.

Kao, D. W.; Kubota, A.; Nevins, M. \& Fiorellini, J. P. The negative effect of combining rhBMP-2 and Bio-Oss on bone formation for maxillary sinus augmentation. Int. J. Periodontics Restorative Dent., 32(1):61-7, 2012.

Kaneko, H.; Arakawa, T.; Mano, H.; Kaneda, T.; Ogasawara, A.; Nakagawa, M.; Toyama, Y.; Yabe, Y.; Kumegawa, M. \& Hakeda, Y. Direct stimulation of osteoclastic bone resorption by bone morphogenetic protein (BMP)-2 and expression of BMP receptors in mature osteoclasts. Bone, 27(4):479-86, 2000.

Klüppel, L. E.; Antonini, F.; Olate, S.; Nascimento, F. F.; Albergaria-Barbosa, J. R. \& Mazzonetto, R. Bone repair is influenced by different particle sizes of anorganic bovine bone matrix: a histologic and radiographic study in vivo. J. Craniofac. Surg., 24(4):1074-7, 2013.
Li, R. H. \& Wozney, J. M. Delivering on the promise of bone morphogenetic proteins. Trends Biotechnol., 19(7):255-65, 2001.

Naujokat, H.; Açil, Y.; Harder, S.; Lipp, M.; Böhrnsen, F. \& Wilfang, J. Osseointegration of dental implants in ectopic engineered bone in three different scaffold materials. Int. J. Oral. Maxillofac. Surg., 2019. In press. Doi: 10.1016/j.ijom.2019.04.005.

Nishimura, I.; Shimizu, Y. \& Ooya, K. Effects of cortical bone perforation on experimental guided bone regeneration. Clin. Oral Implants. Res., 15(3):293300, 2004.

Olate, S.; Vásquez, B.; Sandoval, C.; Vasconcellos, A.; Alister, J. P. \& del Sol, M. Histological analysis of bone repair in mandibular body osteotomy using internal fixation system in three different gaps without bone graft in an animal model. Biomed Res. Int., 2019:8043510, 2019.

Raina, D. B.; Larsson, D.; Mrkonjic, F.; Isaksson, H.; Kumar, A.; Lidgren, L. \& Tägil, M. Gelatin- hydroxyapatite- calcium sulphate based biomaterial for long term sustained delivery of bone morphogenic protein- 2 and zoledronic acid for increased bone formation: In-vitro and in-vivo carrier properties. $J$. Control Release, 272:83-96, 2018.

Schwartz-Arad, D. \& Chaushu, G. Placement of implants into fresh extraction sites: 4 to 7 years retrospective evaluation of 95 immediate implants. $J$. Periodontol., 68(11):1110-6, 1997.

Seeherman, H. J.; Li, X. J.; Bouxsein, M. L. \& Wozney, J. M. rhBMP-2 induces transient bone resorption followed by bone formation in a nonhuman primate core defect model. J. Bone Joint Surg. Am., 92(2):411-26, 2010.

Tovar, N.; Jimbo, R.; Gangolli, R.; Perez, L.; Manne, L.; Yoo, D.; Lorenzoni, F.; Witek, L. \& Coelho, P. G. Evaluation of bone response to various anorganic bovine bone xenografts: an experimental calvaria defect study. Int. J. Oral Maxillofac, Surg., 43(2):251-60, 2014.

Uribe, F.; Cantín, M.; Alister, J. P.; Vilos, C.; Fariña, R. \& Olate, S. Bone morphogenetic protein and its option as an alveolar cleft treatment. Int. J. Morphol., 35(1):310-8, 2017.

Uribe, F.; Alister, J. P.; Zaror, C.; Olate, S. \& Fariña, R. Alveolar cleft reconstruction using morphogenetic protein (rhBMP-2): A systematic review and meta-analysis. Cleft Palate Craniofac. J., 2019. In press. Doi: 10.1177/ 1055665619886142.

Vandeweghe, S.; Leconte, C.; Ono, D.; Coelho, P. G. \& Jimbo, R. Comparison of histological and three-dimensional characteristics of porous titanium granules and deproteinized bovine particulate grafts used for sinus floor augmentation in humans: a pilot study. Implant Dent., 22(4):339-43, 2013.

Wang, H. L. \& Boyapati, L. "PASS" Principles for Predictable Bone Regeneration. Implant Dent., 15(1):1-17, 2006.

Wikesjö, U. M.; Xiropaidis, A. V.; Qahash, M.; Lim, W. H.; Sorensen, R. G.; Rohrer, M. D.; Wozney, J. M. \& Hall, J. Bone formation at recombinant human bone morphogenetic protein-2- coated titanium implants in the posterior mandible (Type II bone) in dogs. J. Clin. Periodontol.,35(11):985-91, 2008a.

Wikesjö, U. M.; Qahash, M.; Polimeni, G.; Susin, C.; Shanaman, R. H.; Rohrer, M. D.; Wozney, J. M. \& Hall, J. Alveolar ridge augmentation using implants coated with recombinant human bone morphogenetic protein-2: histologic observations. J. Clin. Periodontol., 35(11):1001-10, 2008b.

Zeren, K. J. Minimally invasive extraction and immediate implant placement: the preservation of esthetics. Int. J. Periodontics Restorative Dent., 26(2):171-81, 2006.

\section{Dirección para correspondencia: \\ Dra. Bélgica Vásquez \\ Facultad de Ciencias de la Salud \\ Universidad de Tarapacá \\ Arica \\ CHILE}

Email: bvasquezp@uta.cl

Recibido : 15-10-2019

Aceptado: 24-11-2019 